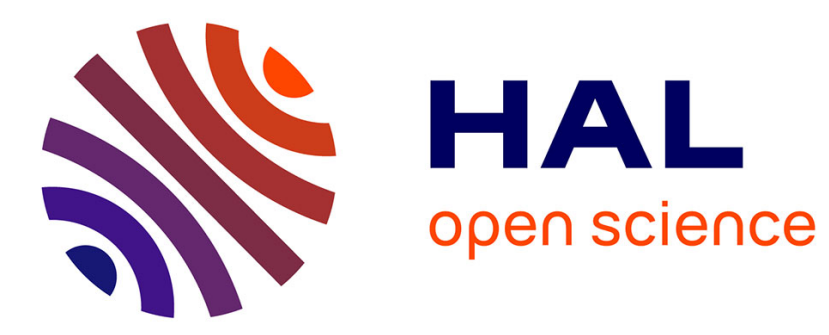

\title{
Qualité diélectrique de couches minces isolantes de GaN obtenues par pulvérisation cathodique réactive \\ Régis Carin
}

\section{To cite this version:}

Régis Carin. Qualité diélectrique de couches minces isolantes de GaN obtenues par pulvérisation cathodique réactive. Revue de Physique Appliquée, 1990, 25 (6), pp.489-497. 10.1051/rphysap:01990002506048900 . jpa-00246211

\section{HAL Id: jpa-00246211 \\ https://hal.science/jpa-00246211}

Submitted on 1 Jan 1990

HAL is a multi-disciplinary open access archive for the deposit and dissemination of scientific research documents, whether they are published or not. The documents may come from teaching and research institutions in France or abroad, or from public or private research centers.
L'archive ouverte pluridisciplinaire HAL, est destinée au dépôt et à la diffusion de documents scientifiques de niveau recherche, publiés ou non, émanant des établissements d'enseignement et de recherche français ou étrangers, des laboratoires publics ou privés. 


\title{
Qualité diélectrique de couches minces isolantes de GaN obtenues par pulvérisation cathodique réactive
}

\author{
R. Carin \\ LERMAT, URA CNRS 1317, ISMRA, Université, 14032 Caen Cedex, France
}

(Reçu le 20 septembre 1989, accepté le 19 février 1990)

\begin{abstract}
Résumé. - Les propriétés diélectriques de couches minces isolantes de $\mathrm{GaN}$, obtenues à $150^{\circ} \mathrm{C}$ par pulvérisation cathodique réactive, sont analysées à température ambiante avec, comme perspectives, la réalisation de structures MIS et la passivation de GaAs. La dispersion de l'indice optique est déterminée par spectrophotométrie. La permittivité, déduite de mesures capacitives, est $\varepsilon_{\mathrm{r}}=12,4 \pm 0,5$ dans le domaine $10^{3}$ $10^{7} \mathrm{~Hz}$; elle présente une dispersion à plus basse fréquence. La conductivité, dominée par l'effet PooleFrenkel, atteint $10^{-13} \Omega^{-1} \mathrm{~cm}^{-1}$ à faible champ. Le champ de claquage est de $1,5 \mathrm{MV} \mathrm{cm}^{-1}$. Une étude comparative de ces propriétés est menée simultanément pour des couches minces de AlN obtenues par le même procédé. Enfin sont analysées les limitations de l'emploi de $\mathrm{GaN}$ comme isolant dans des structures MIS.
\end{abstract}

\begin{abstract}
The dielectric properties of $\mathrm{GaN}$ insulating films grown at $150^{\circ} \mathrm{C}$ by reactive sputtering are investigated for device purposes : realization of MIS structures and passivation of GaAs. The dispersion of the optical index is obtained by reflexion measurements with a spectrophotometer. The permittivity is deduced from capacitive measurements : its value is $\varepsilon_{\mathrm{r}}=12.4 \pm 0.5$ between $10^{3}$ and $10^{7} \mathrm{~Hz}$, and shows a dispersion at lower frequencies. The conductivity is dominated by the Poole-Frenkel effect. Its value can reach $10^{-13} \Omega^{-1} \mathrm{~cm}^{-1}$ at low fields. The breakdown field is around $1.5 \mathrm{MV} \mathrm{cm}{ }^{-1}$. A comparative study is performed for AlN films deposited by a similar process. Finally, the limits of the use of GaN as an insulator for MIS structures are analyzed.
\end{abstract}

\section{Introduction.}

Le nitrure de gallium GaN est un composé semiconducteur III-V à grand gap (direct) qui peut présenter deux sortes d'applications dans le domaine de l'optoélectronique et de la microélectronique. Obtenu sous forme monocristalline et dopé, ce matériau permet de réaliser des jonctions pour fabriquer des diodes électroluminescentes émettant à la limite du violet et de l'UV. En revanche, si l'on produit du GaN suffisamment proche du composé intrinsèque, on peut exploiter ses propriétés d'isolant électrique et sa bonne stabilité chimique pour réaliser des couches diélectriques ou de passivation.

Historiquement, ce sont les applications potentielles de $\mathrm{GaN}$ pour l'électroluminescence qui ont poussé le développement d'études scientifiques sur ce matériau, surtout à partir de 1969: l'objectif principal était la synthèse de monocristaux, qui se heurtait à des difficultés d'ordre technologique [1]. Diverses techniques ont été utilisées et ont montré que c'est surtout la température d'élaboration de $\mathrm{GaN}$ qui semble conditionner son état cristallin : les monocristaux sont obtenus au-dessus de $550^{\circ} \mathrm{C}$, sous forme d'aiguilles de petite dimension (vers $1000^{\circ} \mathrm{C}$, par nitruration de $\mathrm{Ga}$ par $\mathrm{NH}_{3}$ [2] ou par chauffage de poudre $\mathrm{GaN}$ sous $\mathrm{NH}_{3}$ [3]) ou sous forme de couches épitaxiales (par diverses techniques CVD [4-11] et MOCVD [12-14] ou par MBE [15]). Les techniques de dépôt à basse température (moins de $450^{\circ} \mathrm{C}$ ) fournissent en revanche des couches microcristallines: pulvérisation cathodique réactive [1621] et MOCVD-plasma [22, 23]. De plus, la résistivité des échantillons monocristallins non intentionnellement dopés est relativement faible, de l'ordre de $10^{-3}-10^{-1} \Omega \mathrm{cm}$ : le matériau est toujours obtenu dopé de type $\mathrm{n}^{+}$, avec des concentrations de porteurs de $10^{19}$ à $10^{17} \mathrm{~cm}^{-3}$ dont les valeurs plus ou moins élevées semblent corrélées avec celles de la température d'élaboration. Ce résultat est généralement expliqué en invoquant la décomposition thermique de $\mathrm{GaN}$, qui se produit vers $600^{\circ} \mathrm{C}$ : les monocris- 
taux sont obtenus dans des conditions hors-équilibre thermodynamique et contiennent des densités importantes de lacunes d'azote, défauts qui fournissent un niveau donneur peu profond [24]. La principale difficulté pour fabriquer des diodes électroluminescentes est alors la quasi-impossibilité d'obtenir, même par dopage intentionnel, un monocristal GaN de type $\mathrm{p}$ [11].

Les dépôts réalisés à basse température ne présentent pas l'inconvénient de cette décomposition: lorsque les conditions d'élaboration sont convenablement ajustées, on observe pour les couches microcristallines des résistivités comprises entre $10^{8}$ et $10^{13} \Omega \mathrm{cm}$ [16-23]. GaN présente alors un comportement d'isolant électrique, dont la qualité n'est pas aussi bonne que celles des diélectriques couramment utilisés en microélectronique $\left(\mathrm{SiO}_{2}, \mathrm{Si}_{3} \mathrm{~N}_{4}\right)$, mais qui possède d'autres avantages particuliers. Ainsi, on peut s'attendre à une valeur assez élevée de la permittivité diélectrique basse fréquence, compte tenu de la valeur $\varepsilon(0) \simeq 12$ obtenue dans l'infrarouge [3], ce qui est une propriété favorable pour la réalisation de capacités. Enfin, GaN est surtout un composé qui, chimiquement, est assez voisin de l'arséniure de gallium: il peut être produit (très superficiellement) par nitruration ou bombardement à l'azote de substrats de GaAs [10; 25-28]. Comme, par ailleurs, l'étude par XPS de couches minces de GaN [29] a montré que celles-ci assurent une relative imperméabilité à l'oxydation (dont on sait qu'elle est la cause d'une forte activité électrique des surfaces de GaAs), GaN apparaît comme un candidat intéressant pour réaliser une passivation des substrats de GaAs. L'efficacité d'une telle protection nécessite une couche de nitrure suffisamment épaisse, que ne peut fournir la nitruration directe de GaAs : elle pourrait être obtenue par un dépôt de GaN consécutif à la nitruration des substrats.

Dans cet article sont considérées des couches minces isolantes de $\mathrm{GaN}$ obtenues à basse température par pulvérisation cathodique réactive. Leur permittivité diélectrique est analysée dans le domaine optique mais aussi en basse fréquence, domaine pour lequel il n'existe pas de valeur numérique publiée. Leurs caractéristiques électriques (résistivité et claquage) sont étudiées en cherchant à expliquer les mécanismes de conduction et à déterminer les limites du pouvoir d'isolation de $\mathrm{GaN}$ pour des applications orientées vers la réalisation de capacités ou de structures MIS, en particulier sur des substrats de GaAs. Toute cette étude est menée parallèlement pour des couches minces de $\mathrm{GaN}$ et de nitrure d'aluminium AIN réalisées dans des conditions analogues. Ce matériau, qui est assez bien connu [30], est chimiquement et structuralement très voisin de $\mathrm{GaN}$, pour lequel il constitue un bon élément de comparaison, tout en étant un meilleur isolant. Cependant, si l'emploi de AlN a été parfois envisagé pour la réalisation de structures MIS sur GaAs [31, 32], il ne présente pas l'avantage, comme $\mathrm{GaN}$, de pouvoir être produit par nitruration directe de la surface des substrats. Cette dernière propriété, qui permet d'espérer une meilleure passivation électrique de la surface de GaAs à l'aide de $\mathrm{GaN}$, explique l'intérêt potentiel de couches minces isolantes de GaN. Le travail présenté ici se limite à l'étude des couches isolantes déposées sur divers substrats et obtenues, dans le cas de GaAs, sans nitruration préalable. La désoxydation de substrats de GaAs avec production de GaN par bombardement ionique à l'azote a fait l'objet d'une publication antérieure [28]; l'étude des propriétés de l'interface $\mathrm{GaN} / \mathrm{GaAs}$ suite à un tel traitement est en projet.

\section{Elaboration, stœchiométrie et structure des cou- ches de nitrures, préparation des échantillons.}

Les couches minces de nitrures sont obtenues par pulvérisation cathodique réactive d'une cible de gallium ou d'aluminium dans une atmosphère d'azote pur. Le dispositif de dépôt est de type diode radio-fréquence [33]. Un soin particulier est apporté pour limiter les causes de pollution des couches réalisées : emploi de pompes cryogéniques et de gaz de pureté maximale $\left(10^{-6}\right)$. Dans le cas de $\mathrm{GaN}$, les conditions de dépôt ont été optimisées [34] pour obtenir un composé stœchiométrique, en se basant en particulier sur la valeur de l'indice optique des couches produites. Pour une distance inter-électrode de $35 \mathrm{~mm}$, la pression d'azote est ajustée à $1,7 \mathrm{~Pa}$ et la tension d'autopolarisation de la décharge à $2 \mathrm{kV}$. La conduite des dépôts est pratiquement insensible à la nature des substrats : lamelles de quartz, plaquettes de silicium ou de GaAs. La température du porte-échantillon est fixée à $150^{\circ} \mathrm{C}$.

La composition chimique de couches déposées sur Si a été déterminée par RBS et est conforme à la stœchiométrie [33] : en particulier, on ne trouve pas la présence d'oxygène dans le nitrure, résultat qui est confirmé par l'analyse ESCA [29]. La bonne qualité des couches de $\mathrm{GaN}$ est attestée par la valeur de leur seuil d'absorption optique $(3,3 \mathrm{eV})$, déterminée expérimentalement par spectrophotométrie en transmission pour des dépôts sur quartz [33] ; ceci est conforme aux valeurs couramment obtenues pour les échantillons monocristallins [1]. En ce qui concerne $\mathrm{AlN}$, le seuil est obtenu à $5,8 \mathrm{eV}$ en accord avec la plupart des résultats obtenus pour des couches minces [30].

Les monocristaux de GaN et AlN présentent une structure hexagonale (type wurtzite) faiblement anisotropique. L'étude des couches minces par microscopie électronique [35] a montré que ces dépôts réalisés à basse température sont microcristallins et présentent une structure colonnaire; les grains colonnaires, dont le diamètre est de l'ordre de 
la dizaine de $\mathrm{nm}$, sont perpendiculaires à la surface du substrat et à la couche mince; leur croissance s'est faite parallèlement à l'axe c de la structure hexagonale du nitrure.

Les échantillons étudiés sont réalisés à partir de plaquettes polies de Si et GaAs dont le dopage est de l'ordre de $10^{16} \mathrm{~cm}^{-3}$ (types $\mathrm{n}$ et $\mathrm{p}$ ) ou supérieur à $10^{18} \mathrm{~cm}^{-3}$ (type $\mathrm{n}^{+}$). La réflectance des plaques nues varie de $30 \%$ à $60 \%$ environ dans le domaine visible et l'UV proche. Les couches minces de nitrures ont des épaisseurs de quelques centaines de $\mathrm{nm}$ avec une précision de 2 à $3 \%$ (mesures par ellipsométrie, à $\lambda=632,8 \mathrm{~nm}$ ). Pour effectuer les mesures électriques, on réalise des structures MIS: des grilles d'aluminium de quelques dizaines de $\mathrm{nm}$ d'épaisseur sont évaporées thermiquement sur le nitrure, à travers un masque constitué d'une plaque métallique percée de trous circulaires de différentes dimensions ; pendant l'évaporation, le masque est placé à moins de $1 \mathrm{~mm}$ de la surface de l'échantillon, et le filament d'Al est situé à $12 \mathrm{~cm}$, si bien que les plots évaporés reproduisent le dessin des trous avec une précision meilleure que $1 \%$. Les diamètres des trous du masque ont été mesurés au microscope à l'aide d'un réticule gradué ; leurs surfaces $S$ sont respectivement $1,2 \mathrm{~mm}^{2} ; 2,5 \mathrm{~mm}^{2} ; 5,7 \mathrm{~mm}^{2}$ (avec une incertitude de l'ordre de $1 \%$ pour les plus grands). Les substrats sont munis de contacts ohmiques sur leurs faces arrières.

\section{Indice optique et permittivité diélectrique.}

La dispersion de l'indice optique $n_{0}$ des couches de nitrures est obtenue par spectrophotométrie en réflexion. Les spectres de réflectance des échantillons, obtenus en incidence normale (champ électrique $\mathbf{E} \perp \mathbf{c}$ ), présentent des oscillations dues aux interférences entre les faces de la lame mince de nitrure. L'épaisseur de celle-ci étant connue, l'exploitation des positions en longueur d'onde $\lambda$ des extrema d'interférence permet de déduire les valeurs correspondantes de $n_{0}$. La figure 1 fournit des résultats représentatifs des divers échantillons étudiés. On observe que, de façon tout à fait classique, $n_{0}$ croît de manière sensible au voisinage des seuils d'absorption; les valeurs de $n_{0}$ dans le domaine visible situé loin de la zone d'absorption, 1,95 pour AlN et 2,35 pour GaN, sont cohérentes avec les mesures effectuées par ellipsométrie à $\lambda=632,8 \mathrm{~nm}$. Dans le cas de $\mathrm{GaN}$, la courbe de dispersion dans son ensemble est conforme à celle obtenue pour un monocristal [2], et en accord avec la valeur précise $n_{0}=2,397$ déterminée par ellipsométrie à $\lambda=546,1 \mathrm{~nm}$ pour une couche épitaxiée [6].

La permittivité diélectrique $\varepsilon_{\mathrm{r}}$ des nitrures est déterminée à partir de mesures capacitives $(\mathbf{E} / / \mathbf{c})$ effectuées à diverses fréquences $(10 \mathrm{~Hz}-10 \mathrm{MHz})$ sur

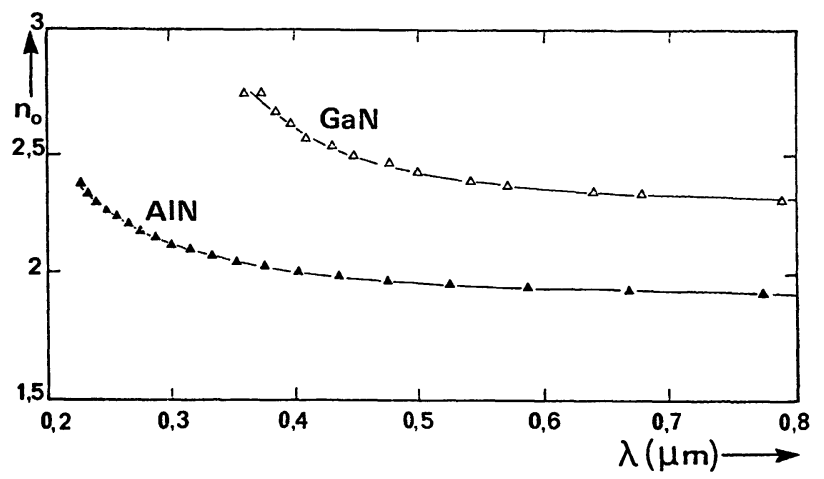

Fig. 1. - Dispersion de l'indice optique avec la longueur d'onde, pour des couches minces microcristallines de $\mathrm{GaN}$ et $\mathrm{AlN}$ (avec $\mathbf{E} \perp \mathbf{c})$.

[Refractive index versus wavelength, at $300 \mathrm{~K}$, for $\mathrm{GaN}$ and AIN microcrystalline films $(\mathbf{E} \perp \mathbf{c})$.]

les structures MIS obtenues avec des substrats de type $\mathrm{n}^{+}$[33]. Les valeurs de capacités étaient mesurées en situation de complète accumulation des structures métal/nitrure/Si- $n^{+}$: aucun effet de désertion de la surface du semi-conducteur (qui aurait pu diminuer la capacité, dans le cas d'un substrat $\mathrm{n}^{+}$, de seulement quelques \%) ne faussait les mesures. Dans ces conditions, l'incertitude sur les valeurs de $\varepsilon_{\mathrm{r}}$ est inférieure à $5 \%$, sauf dans le domaine haute fréquence (3-10 MHz) où le dispositif de mesure capacitive, sensible à certaines capacités parasites, introduit des erreurs de l'ordre de $10 \%$. Les résultats obtenus sont représentés sur la figure 2 .

Pour AlN, on n'observe pas de dispersion de la permittivité avec la fréquence et la valeur $\varepsilon_{\mathrm{r}}=$ $8,5 \pm 0,3$ est parmi les plus fréquemment obtenues pour ce composé [30]. Pour $\mathrm{GaN}$, on observe à basse fréquence une dispersion de type relaxation dipolaire : une telle dispersion a déjà été observée de manière plus accentuée que dans notre cas pour des couches minces de $\mathrm{GaN}$ déposées également par pulvérisation cathodique réactive; ce phénomène a été expliqué par la présence probable d'ions gallium en excès [19]. Un travail complémentaire du précédent donne des valeurs de $\varepsilon_{\mathrm{r}}$ de l'ordre de 15 à $100 \mathrm{kHz}$ [23] et étudie plus précisément la composition chimique de couches minces obtenues pour différentes conditions de dépôt par pulvérisation cathodique réactive et par CVD-plasma : elle montre que les résultats précédents concernent en fait de l'oxynitrure de gallium pouvant contenir jusqu'à $60 \%$ d'oxygène par rapport à l'azote ; il est en outre suggéré que l'incorporation d'oxygène peut réduire la dispersion de $\varepsilon_{\mathrm{r}}$, mais ce résultat peut s'expliquer par l'élimination des lacunes d'azote et pourrait aussi être obtenu pour GaN stœchiométrique. Une autre étude a d'ailleurs montré que cette dispersion pouvait être diminuée (voire éliminée) si le dépôt par pulvérisation cathodique réactive était effectué à 


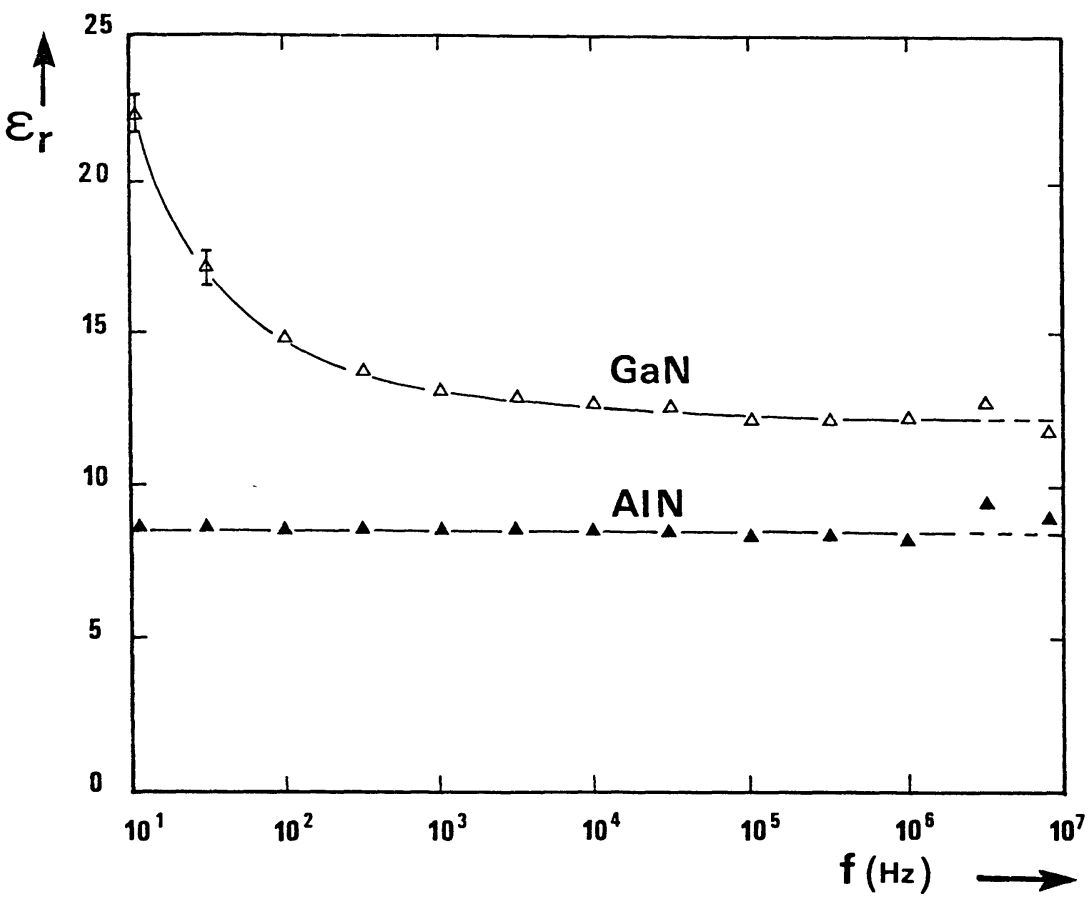

Fig. 2. - Permittivité diélectrique en fonction de la fréquence, pour des couches minces de GaN et $\mathrm{AlN}$ (avec E// c).

[Dielectric permittivity versus frequency, at $300 \mathrm{~K}$, for $\mathrm{GaN}$ and $\mathrm{AlN}$ films (E//c).]

$350^{\circ} \mathrm{C}$ ou si l'on procédait à un recuit sous hydrogène [36].

La valeur atteinte au-delà du domaine de retaxation dipolaire de $\mathrm{GaN}$ (Fig. 2) est $\varepsilon_{\mathrm{r}}=12,4 \pm 0,5$ : à notre connaissance, il s'agit là de la première mesure précise de cette grandeur physique dans ce domaine de fréquence; on peut la comparer aux valeurs obtenues par des mesures de réflectivité infra-rouge sur des monocristaux (pour E//c) $\varepsilon(0)=12 \pm 2$ [3] et $\varepsilon(0)=10,4 \pm 0,3$ [9] : l'accord est raisonnable si l'on tient compte d'une légère dispersion entre ces deux domaines de fréquence (entre $10^{6}$ et $10^{13} \mathrm{~Hz}$ ). Cette valeur relativement élevée de $\varepsilon_{\mathrm{r}}$ dans $\mathrm{GaN}$ est proche de celles des semi-conducteurs à liaisons tétraédriques ( $\mathrm{Si}, \mathrm{GaAs})$ : dans le cas d'un matériau destiné à l'isolation électrique de structures MIS, une valeur aussi grande de $\varepsilon_{\mathrm{r}}$ peut être un avantage car elle permet, pour une épaisseur donnée de la couche d'isolant, d'obtenir des valeurs plus importantes de la capacité.

\section{Conductivité électrique.}

4.1 RÉSUltats EXPÉRIMENTAUX. - La conductivité $\sigma$ des nitrures est déduite simplement des caractéristiques $I-V$ en continu des échantillons :

$$
\sigma=\mathrm{d} I / S V
$$

Le faible courant de fuite $I$ traversant la mince couche de nitrure, constituant l'isolant de la structure
MIS, est mesuré avec un électromètre KEITHLEY 617, qui est capable de déceler une fraction de pA. La tension $V$ appliquée à la grille est mesurée avec un montage type "longue dérivation" aux bornes de l'ensemble MIS-électromètre, placés en série.

La relation (1) suppose que la tension $V$ appliquée extérieurement se retrouve entre les faces de la couche mince, ce qui nécessite l'absence de zone désertée dans le semi-conducteur: cette condition est pratiquement toujours vérifiée pour les échantillons réalisés sur des substrats de type $\mathrm{n}^{+}$. Dans leur cas, les caractéristiques $I-V$ obtenues expérimentalement ne sont pas linéaires : aux tensions élevées, le courant croît de façon quasi exponentielle avec $V$. Ce n'est pas un effet de jonction (localisé à l'interface $\mathrm{Al}$ /nitrure ou nitrure/semi-conducteur, ou encore dû au contact arrière sur le substrat) qui peut expliquer ce résultat car les caractéristiques sont pratiquement symétriques entre tensions de grille positives ou négatives. Le mécanisme de conduction correspond à un courant limité en volume et non en surface (au niveau des électrodes). En outre, la variation de $I$ avec $V$ est d'autant plus rapide que la couche de nitrure est plus mince, ce qui suggère pour les fortes tensions un mécanisme en émission de champ électrique $E$, avec:

$$
E=V / d .
$$

Les résultats expérimentaux sont exploités sous forme de courbes $\lg \sigma-\sqrt{E}$ : la figure 3 donne 


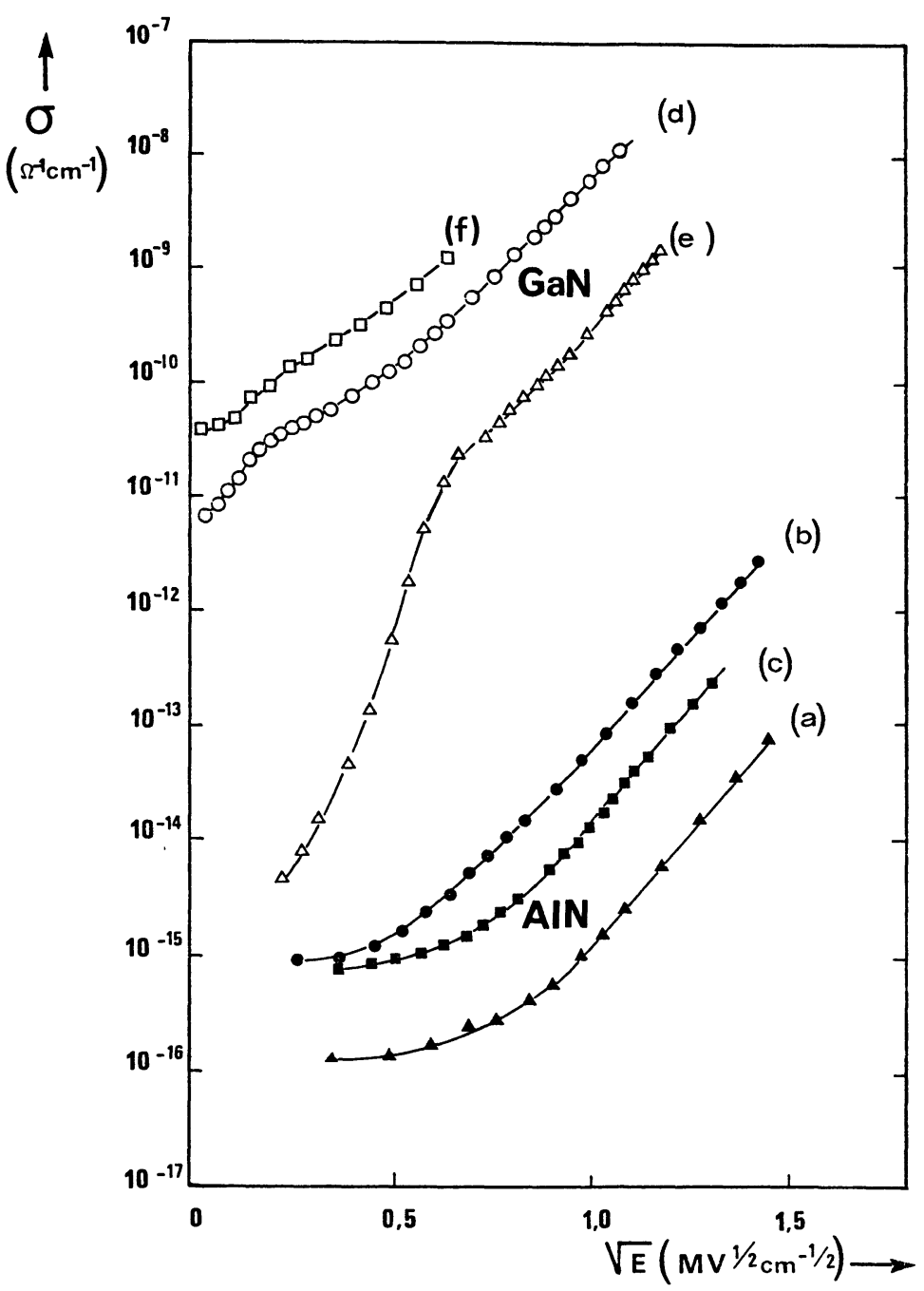

Fig. 3. - Conductivité $\sigma$ en fonction de la racine carrée du champ électrique $E$, pour des couches minces de nitrures pulvérisés : (a) AlN-85 nm/Si-n ${ }^{+}$, (b) AlN-145 nm/Si-n ${ }^{+}$, (c) AlN-150 nm/GaAs-n, (d) GaN-240 nm $/ \mathrm{Si}^{+} \mathrm{n}^{+}$, (e) GaN$200 \mathrm{~nm} / \mathrm{Si}-\mathrm{p}$, (f) GaN-210 nm/GaAs-n.

[Conductivity versus electric field square root, at $300 \mathrm{~K}$, for sputtered films.]

quelques exemples représentatifs à la fois de l'allure des courbes et de la dispersion des résultats. Pour chaque échantillon, la courbe $\lg \sigma-\sqrt{E}$ est identique pour les diverses structures MIS analysées, avec des surfaces de grilles identiques ou différentes $\left(S=1,2-5,7 \mathrm{~mm}^{2}\right)$ : ceci indique la bonne homogénéité de chaque couche de nitrure et montre que la dispersion observée correspond à des dépôts différents. Les courbes obtenues pour des substrats de type $\mathrm{n}^{+}$(Figs. 3a, b, d) sont indifférentes au signe de $V$. En revanche, pour les substrats de type $\mathrm{n}$ et $\mathrm{p}$, on a observé une dissymétrie suivant que la structure MIS était polarisée en direct, vers l'accumulation de porteurs majoritaires à l'interface $(V>0$ pour type $\mathrm{n} ; V<0$ pour type p) ou en inverse ; les courbes représentées (Figs. 3c, e, f) correspondent à la polarisation directe. Celles obtenues en polarisation inverse ne sont pas considérées car leur comportement est fortement influencé par l'existence d'une zone désertée largement développée dans le semiconducteur : suivant le cas, le courant $I$ peut être limité par les phénomènes de génération-recombinaison dans cette zone, sauf à fort champ où elle est le siège de phénomènes d'avalanche; en tous les cas, la relation (2) n'est alors pas valable.

\subsection{TYPE ET MÉCANISME DE CONDUCTION DANS LES} NITRURES. - Dans le cas de GaN, les courbes $\sigma-\sqrt{E}$ ont une allure différente entre les dépôts réalisés sur substrats de type $\mathrm{p}$ (Fig. 3e) et de type $n$ (Fig. 3f) : alors qu'à fort champ l'allure est analogue, les conductivités mesurées à faible champ sont beaucoup plus faibles pour le type $p$ que pour le type $\mathrm{n}$, indiquant une limitation du courant extérieure au nitrure. Ce résultat, observé en régime de faible tension où la surface du semi-conducteur est faiblement désertée (à cause de la présence d'états d'interface chargés), suggère une conduction du nitrure de 
type $\mathrm{n}$ : en effet, pour les substrats $\mathrm{p}$ (Fig. 3e, $V<0$ ), le courant d'électrons est alors limité par la recombinaison avec les trous dont la densité est faible dans la zone désertée du semi-conducteur; pour les substrats n (Fig. 3f, $V>0$ ), la zone désertée est le siège d'une simple diffusion électronique et la limitation du courant est due à la résistivité du nitrure comme dans le cas des substrats fortement dopés.

A fort champ électrique, la variation de $\lg \sigma$ avec $\sqrt{E}$ est linéaire à la fois pour AIN et GaN (Fig. 3), ce qui peut être expliqué par un mécanisme de conduction des nitrures de type Poole-Frenkel. Ce mécanisme suppose l'existence dans le matériau de centres donneurs profonds, qui peuvent être dus à des impuretés et qui se comportent en centres coulombiens vis-à-vis des électrons émis. A faible champ, l'émission est possible seulement vers des niveaux-pièges localisés dans la bande interdite, qui peuvent être dus au désordre (pour les couches microcristallines, la densité de tels états doit être importante au voisinage des joints de grains), entre lesquels se produit une conduction par sauts de type. ohmique. Un fort champ électrique réduit notablement la hauteur de la barrière de potentiel qui commande l'émission vers la bande de conduction de l'isolant; celle-ci se produit, à température ambiante, principalement par excitation thermique [37] et la conductivité Poole-Frenkel s'écrit :

$$
\sigma_{\mathrm{PF}}=\sigma_{0} \exp \left[-\left[\phi-q \sqrt{q E / \pi \varepsilon \varepsilon_{0}}\right] / k T\right]
$$

où $\sigma_{0}$ est proportionnel à la densité des centres donneurs, $\phi$ est la profondeur en énergie du niveau donneur par rapport au bas de la bande de conduction. $\varepsilon$ est la permittivité de l'isolant et traduit la réponse dynamique du diélectrique lors de la libération du porteur: elle se situe entre $\varepsilon(\infty)$ et $\varepsilon(0)$ suivant le matériau et peut présenter des valeurs apparentes plus élevées lorsque les centres coulombiens sont susceptibles d'interagir [38].

En utilisant l'équation (3), la partie linéaire à haut champ des courbes expérimentales $\lg \sigma-\sqrt{E}$ (Fig. 3) a permis de déterminer, indépendamment de l'épaisseur des couches, les valeurs $\varepsilon=11$ pour AlN et $\varepsilon=13$ pour GaN. Celles-ci sont proches des valeurs de $\varepsilon_{\mathrm{r}}$ obtenues par mesures capacitives sur ces mêmes couches, donc des valeurs de $\varepsilon(0)$ : l'écart est assez faible pour que l'on puisse expliquer de façon cohérente la conductivité de ces nitrures par un effet Poole-Frenkel classique intervenant à partir de centres coulombiens isolés.

La conductivité Poole-Frenkel résiduelle à bas champ $\sigma_{0} \exp (-\phi / k T)$ est nettement plus faible pour AlN $\left(10^{-18}-10^{-17} \Omega^{-1} \mathrm{~cm}^{-1}\right)$ que pour $\mathrm{GaN}$ $\left(10^{-13}-10^{-11} \Omega^{-1} \mathrm{~cm}^{-1}\right)$, ce qui traduit vraisemblablement l'effet de niveaux donneurs situés plus profondément plutôt qu'une densité de centres beau- coup plus faible: une étude en fonction de la température devrait permettre de conclure. Pour chaque nitrure, la dispersion des valeurs de cette conductivité résiduelle n'est pas corrélée à l'épaisseur des couches ou aux conditions de dépôt : d'amplitude relativement faible, cette dispersion est peut-être due à des fluctuations de $\sigma_{0}$ (densité de centres donneurs) ou à une réorganisation différente des centres sous l'effet du champ électrique lors des premières mises sous tension des différentes structures.

Pour AlN, on observe à faible champ une conductivité de type ohmique (Fig. 3) dont la valeur $\left(10^{-16}-10^{-15} \Omega^{-1} \mathrm{~cm}^{-1}\right)$ traduit - l'importance des états-pièges localisés. Si l'on admet que ceux-ci sont surtout dus au désordre de la structure microcristalline, il est vraisemblable que les couches de $\mathrm{GaN}$ sont le siège d'un phénomène analogue qui se trouve masqué par la conduction Poole-Frenkel résiduelle à bas champ. Dans le cas de GaN, on peut relever une ondulation de la courbe $\lg \sigma-\sqrt{E}$ à faible champ (Fig. 3d). Celle-ci s'explique peut-être par un phénomène supplémentaire de courant émis en charge d'espace avec remplissage d'un niveau-piège : de tels phénomènes ont déjà été observés pour des couches de $\mathrm{GaN}$ de quelques $\mu \mathrm{m}$ d'épaisseur [17] (des couches environ dix fois plus épaisses que les nôtres permettent une étude plus détaillée du domaine de faible champ électrique). Une autre explication possible de cette ondulation est la migration d'ions inclus dans le nitrure sous l'effet du champ électrique. Cette dernière explication est cohérente avec l'observation suivante: lorsque l'on applique, pour la première fois après sa fabrication, une tension $V$ sur la grille d'une structure $\mathrm{Al} / \mathrm{GaN} / \mathrm{Si}-\mathrm{n}^{+}$, on obtient un courant $I$ qui diminue lentement avec le temps; l'application progressive de tensions plus élevées conduit à une stabilisation des caractéristiques $I-V$; il est vraisemblable que la couche de nitrure pulvérisé doit contenir lors de son élaboration de nombreux défauts et impuretés ionisés (susceptibles de contribuer comme centres coulombiens à la conduction Poole-Frenkel) qui, sous l'action du champ électrique appliqué, se réorganisent partiellement.

\subsection{Claquage Des COUChes minces. -} L'augmentation brutale de la conductivité pour un fort champ électrique peut s'expliquer par la rigidité diélectrique du matériau ou par des phénomènes d'avalanche de porteurs. Dans le cas de nos couches de nitrures déposés par pulvérisation cathodique réactive, ce claquage se produit de façon indépendante de l'épaisseur à des champs de l'ordre de $2 \mathrm{MV} \mathrm{cm}^{-1}$ pour AlN et $1,5 \mathrm{MV} \mathrm{cm}^{-1}$ pour GaN. Ces valeurs sont trop faibles pour refléter la rigidité diélectrique, c'est-à-dire le champ nécessaire pour briser les liaisons chimiques dans le matériau (de 
l'ordre de $10 \mathrm{MV} \mathrm{cm}^{-1}$ dans $\mathrm{SiO}_{2}$ ou $\mathrm{Si}_{3} \mathrm{~N}_{4}$ amorphe [39]). Deux explications sont possibles: soit la structure polycristalline colonnaire des couches minces produit le long des joints de grains des chemins privilégiés de fragilisation des couches, soit le claquage est dû à un effet d'ionisation et d'avalanche de création de porteurs dans les grains de nitrure cristallin, par impact à partir des porteurs primaires dus à l'effet Poole-Frenkel (des effets d'avalanche sont observés dans $\mathrm{Si}$ ou GaAs cristallisé pour des champs de 0,3 à $0,4 \mathrm{MV} \mathrm{cm}^{-1}$ [39]). Ces claquages se produisent à des champs qui sont néanmoins suffisamment élevés pour que les structures MIS puissent supporter des tensions supérieures à $20 \mathrm{~V}$ pour des épaisseurs de $150 \mathrm{~nm}$ de AlN ou de $200 \mathrm{~nm}$ de GaN.

\section{Perspectives d'utilisation de GaN comme isolant dans des dispositifs MIS sur GaAs.}

Les résistivités des couches minces microcristallines présentées dans ce travail figurent parmi les valeurs les plus élevées obtenues à ce jour à la fois pour AlN $\left(10^{11}-10^{16} \Omega \mathrm{cm}\right.$ [30]) et pour GaN $\left(10^{8}\right.$ $10^{13} \Omega \mathrm{cm}$ [16-23]), ce qui montre la bonne qualité du procédé d'élaboration. Les propriétés diélectriques observées sont sans doute moins favorables que celles des dépôts de $\mathrm{Si}_{3} \mathrm{~N}_{4}$ amorphe couramment utilisés en microélectronique. Néanmoins, pour GaN, la permittivité plus élevée permet d'obtenir des valeurs de capacités convenables avec des couches plus épaisses, ce qui permet de compenser partiellement l'effet de la résistivité plus faible. La qualité des couches isolantes est suffisante pour réaliser et expérimenter des structures MIS, en particulier sur GaAs.

La figure 4 représente les caractéristiques $C-V$ obtenues à différentes fréquences pour une telle structure réalisée sur un substrat de GaAs de type n (dopé à $10^{16} \mathrm{~cm}^{-3}$ ). L'allure générale de ce réseau de courbes, qui présente en particulier une dispersion importante pour les polarisations positives, est similaire à ce qui a déjà été observé pour des structures $\mathrm{Al} / \mathrm{Si}_{3} \mathrm{~N}_{4} / \mathrm{GaAs}-\mathrm{n}$ [40] : elle s'explique par la présence, à l'interface isolant/semi-conducteur, de densités importantes $\left(\sim 10^{13} \mathrm{eV}^{-1} \mathrm{~cm}^{-2}\right)$ d'états localisés situés dans la bande interdite de GaAs. Ces états d'interface sont analogues à ceux que l'on observe après oxydation de substrats de GaAs [41], celle-ci induisant à la surface du semi-conducteur III-V de

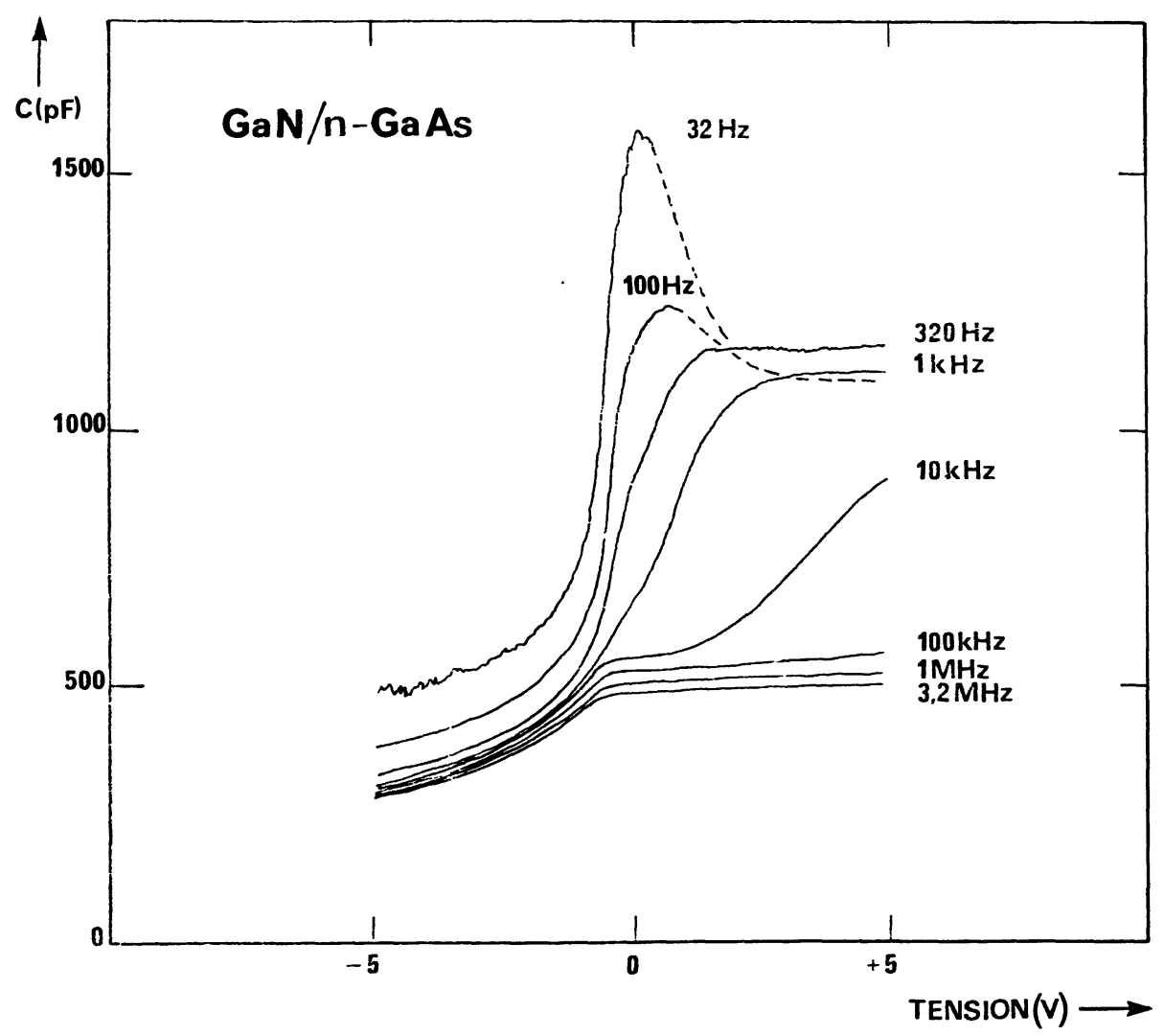

Fig. 4. - Caractéristiques $C-V$ d'une structure MIS Al/GaN/GaAs-n (dopage $\sim 10^{16} \mathrm{~cm}^{-3} ; d=210 \mathrm{~nm}$; $S=2,5 \mathrm{~mm}^{2}$ ) pour diverses fréquences, avec une vitesse de balayage de la tension de $0,2 \mathrm{~V} \mathrm{~s}^{-1}$.

[Capacitance versus voltage characteristics, for several frequencies, of a MIS structure Al/GaN/GaAs. The doping of the substrate is n-type $\left(\sim 10^{16} \mathrm{~cm}^{-3}\right)$, the nitride thickness is $210 \mathrm{~nm}$, the gate area is $2.5 \mathrm{~mm}^{2}$ and the sweep rate is $0.2 \mathrm{~V} \mathrm{~s}^{-1}$.] 
nombreux défauts de stœchiométrie et de structure [42]. Dans le cas des échantillons nitrure/GaAs, les états d'interface sont certainement dus à l'existence d'oxyde natif à la surface des substrats avant dépôt : la présence de $\mathrm{Ga}_{2} \mathrm{O}_{3}$ à l'interface a d'ailleurs été montrée par une étude par XPS des échantillons GaN/GaAs [29] et justifie la recherche de procédés de désoxydation et de passivation de GaAs [28]. En l'absence de l'emploi de tels procédés pour ces échantillons, l'étude de l'interface ne sera pas ici approfondie. En revanche, on va s'intéresser aux incidences, sur le comportement de la structure MIS, de l'utilisation de GaN comme isolant.

Pour les tensions négatives, on a observé que, dans le cas des structures $\mathrm{Al} / \mathrm{Si}_{3} \mathrm{~N}_{4} / \mathrm{GaAs}-n$, la surface du semi-conducteur se trouve en situation d'inversion, ce qui se traduit dans le réseau de courbes $C-V$ par un palier horizontal identique pour toutes les fréquences [40]. Dans le cas des échantillons $\mathrm{Al} / \mathrm{GaN} / \mathrm{GaAs}-\mathrm{n}$, un tel palier, qui devrait se situer théoriquement vers $C \simeq 450 \mathrm{pF}$ sur la figure 4 , n'est pas obtenu: on observe au contraire une dispersion de la capacité avec la fréquence et des valeurs de capacité qui peuvent être inférieures à celle du palier théorique. La dispersion avec la fréquence pour $V<0$ est facile à interpréter car elle traduit le comportement de la permittivité de GaN observé dans la même gamme de fréquences pour les substrats $\mathrm{Si}^{+} \mathbf{n}^{+}$. Les capacités inférieures au palier indiquent que la surface du semi-conducteur esthors-équilibre [39]: l'alimentation en trous de la couche d'inversion de la structure MIS par les phénomènes de génération de paires électron-trou est vraisemblablement perturbée et la zone désertée est alors plus étendue qu'à l'équilibre (régime de déplétion profonde). La cause de la perturbation est facile à identifier : le courant de fuite de la couche de $\mathrm{GaN}$ (de l'ordre de $10^{2} \mathrm{nA}$ à $-5 \mathrm{~V}$ pour l'échantillon de la Fig. 4) est bien supérieur au courant de génération théorique (environ $0,1 \mathrm{nA} / \mathrm{mm}^{2}$ pour GaAs) et l'injection d'électrons en excès qui en résulte empêche la génération des trous.

Une autre conséquence de la conductivité non négligeable des couches de GaN peut être observée sur les caractéristiques $C-V$ des structures MIS.
Alors que dans le schéma électrique d'une structure MIS idéale, l'isolant apparaît sous la forme d'un condensateur, on doit lui adjoindre une résistance en parallèle dès que la conductance d'isolant n'est plus négligeable par rapport à sa susceptance, c'està-dire si $\sigma \geqslant \varepsilon_{\mathrm{r}} \varepsilon_{0} f$. Compte tenu des résultats expérimentaux précédents (Figs. 2 et 3 ), on peut évaluer à environ $100 \mathrm{~Hz}$ la fréquence limite qui réalise cette condition pour GaN. L'effet de la conductance d'isolant peut être observé sur la figure 4 sous la forme d'un comportement anormal des courbes $C-V$ obtenues aux plus basses fréquences : l'anomalie apparaît surtout pour les polarisations $V>0$ (qui correspondent à une zone désertée étroite et à des valeurs de $E$, donc de $\sigma$, plus élevées), elle se traduit par une diminution apparente de $C$ vers l'accumulation (portions de courbes en trait interrompu) due à l'incapacité du dispositif de mesure à déterminer correctement la susceptance de la structure en présence d'un fort signal de conductance parallèle. Une telle anomalie n'est jamais observée avec de bons isolants tels que $\mathrm{Si}_{3} \mathrm{~N}_{4}$ ou même AlN.

\section{Conclusion.}

Lorsqu'il est élaboré à basse température, le nitrure de gallium $\mathrm{GaN}$, obtenu sous forme microcristalline, est isolant et possède des propriétés diélectriques intéressantes. La permittivité diélectrique est assez élevée : $\varepsilon_{\mathrm{r}} \equiv 12,4$ (vèrs $100 \mathrm{kHz}$ ). La résistivité à faible champ peut atteindre $10^{13} \Omega \mathrm{cm}$, mais on observe une augmentation de la conductivité avec le champ, due à un effet Poole-Frenkel. Le champ de claquage, relativement élevé, atteint $1,5 \mathrm{MV} \mathrm{cm}^{-1}$. L'emploi de GaN comme isolant de structures MIS se heurte à certaines difficultés : le courant de fuite empêche la formation d'une couche d'inversion, et l'on observe en basse fréquence une dispersion de la permittivité et une influence trop grande de la conductance d'isolant par rapport à sa susceptance. Ces résultats laissent néanmoins ouvertes les perspectives d'utilisation de $\mathrm{GaN}$ soit dans des couches isolantes multiples, soit pour un rôle de simple passivation de surfaces de GaAs préalablement désoxydées et nitrurées.

\section{Bibliographie}

[1] Kesamanly F. P., Sov. Phys. Semicond. 8 (1974) 147.

[2] EJder E., Phys. Status Solidi (a) 6 (1971) 445.

[3] Manchon D. D., Barker A. S., Dean P. J. et Zetterstrom R. B., Solid State Commun. 8 (1970) 1227.

[4] Kosicki B. B. et Kahng D., J. Vac. Sci. Technol. 6 (1969) 593.

[5] Maruska H. P. et Tietjen J. J., Appl. Phys. Lett. 15 (1969) 327.
[6] Pankove J. I., Berkeyheiser J. E., Maruska H.P. et Wittke J., Solid State Commun. 8 (1970) 1051.

[7] CHU T. L., J. Electrochem. Soc. 118 (1971) 1200.

[8] Ilegems M., J. Cryst. Growth 13/14 (1972) 360.

[9] Barker A. S. et Ilegems M., Phys. Rev. B 7 (1973) 743.

[10] Lappa R., Glowacki G. et GalkowsKi S., Thin Solid Films 32 (1976) 73. 
[11] JACOB G. et BoIS D., Appl. Phys. Lett. 30 (1977) 412.

[12] Khan M. A., Skogman R. A., Schulze R. G. et Gershenzon M., Appl. Phys. Lett. 42 (1983) 430.

[13] Zembutsu S. et Sasaki T., Appl. Phys. Lett. 48 (1986) 870.

[14] Amano H., Hiramatsu K. et Akasaki I., Jpn J. Appl. Phys. 27 (1988) L1384.

[15] Gotoh H., Suga T., Suzuki H. et Kimata M., Jpn J. Appl. Phys. 20 (1981) L545.

[16] Hovel H. J. et Cuomo J. J., Appl. Phys. Lett. 20 (1972) 71.

[17] Vesely J. C., Shatzkes M. et Burkhardt P. J., Phys. Rev. B 10 (1974) 582.

[18] Puychevrier N. et Menoret M., Thin Solid Films 36 (1976) 141.

[19] Hariu T., Usuba T., Adachi H. et Shibata Y., Appl. Phys. Lett. 32 (1978) 252.

[20] Lakshmi E., Mathur B., Bhattacharyya A. B. et Bhargava V. P., Thin Solid Films 74 (1980) 77.

[21] Zembutsu S. et Kobayashi M., Thin Solid Films 129 (1985) 289.

[22] Knights J. C. et Lujan R. A., J. Appl. Phys. 49 (1978) 1291.

[23] Matsushita K., Matsuno Y., Hariu T. et Shibata Y., Thin Solid Films 80 (1981) 243.

[24] Pankove J. I., Bloom S. et Harbeke G., $R C A$ Rev. 36 (1975) 163.

[25] Pankove J. I., Berkeyheiser J. E., Kilpatrick S. J. et MAgeE C. W., J. Electr. Mater. 12 (1983) 359.

[26] Gourrier S., Smit L., Friedel P. et LARSen P. K., J. Appl. Phys. 54 (1983) 3993.

[27] Alnot P., Grattepain C., Huber A., Wyczisk F., Bourgoin J., Vuillaume D., Joubart R. et
Peray J. F., Vide-Couches Minces 43 (1988) 287.

[28] Carin R., Le Moël A. et Duraud J. P., J. Appl. Phys. 65 (1989) 5051.

[29] Carin R., Deville J. P. et WerckmanN J., Eur. Conf. Applications of Surface and Interface Analysis, ECASIA (Antibes, France) 23-27 octobre 1989, Surf. Interface Anal. (1990) sous presse.

[30] Pauleau Y., Hantzpergue J. J. et Remy J. C., Bull. Soc. Chim. Fr. 5-6 (1979) I199.

[31] Alexandre F., Masson J. M., Post G. et ScavenNEC A., Thin Solid Films 98 (1982) 75.

[32] Fujieda S., Mizuta M. et Matsumoto Y., Jpn J. Appl. Phys. 27 (1988) L296.

[33] Carin R., Thèse de Doctorat ès Sciences, Caen (1989).

[34] Carin R. et Vizot J., C. R. du $4^{\text {e }}$ Colloq. Int. sur les plasmas et la pulvérisation cathodique (13-17 septembre 1982, Nice) Vide-Couches Minces Suppl. 37 (1982) 157.

[35] Martin C., Vizot J., Hervieu M. et Mercey B., IIIrd Eur. Conf. on Solid State Chemistry, Regensburg (29-31 mars 1986) Book of Abstracts, vol. 2, 366 .

[36] LaKshmi E., Thin Solid Films 83 (1981) L137.

[37] HILl R. M., Thin Solid Films 12 (1972) 367.

[38] Hill R. M., Thin Solid Films 8 (1971) R21.

[39] SzE S. M., Physics of Semiconductor Devices, 2nd edition (Wiley) 1981.

[40] Bogdanski P., Carin R., Gardais O. et Vizot J., Phys. Status Solidi (a) 80 (1983) 185.

[41] Sawada T. et Hasegawa H., Thin Solid Films 56 (1979) 183.

[42] Spicer W. E., Lindau I., Skeath P. et Su C. Y., $J$. Vac. Sci. Technol. 17 (1980) 1019. 